\title{
Towards a feasible model for shared decision making: focus group study with general practice registrars
}

\author{
Glyn Elwyn, Adrian Edwards, Richard Gwyn, Richard Grol
}

\begin{abstract}
Objectives To explore the views of general practice registrars about involving patients in decisions and to assess the feasibility of using the shared decision making model by means of simulated general practice consultations.

Design Qualitative study based on focus group interviews.

Setting General practice vocational training schemes in south Wales.

Participants 39 general practice registrars and eight course organisers (acting as observers) attended four sessions; three simulated patients attended each time. Method After an introduction to the principles and suggested stages of shared decision making the registrars conducted and observed a series of consultations about choices of treatment with simulated patients using verbal, numerical, and graphical data formats. Reactions were elicited by using focus group interviews after each consultation and content analysis undertaken.

Results Registrars in general practice report not being trained in the skills required to involve patients in clinical decisions. They had a wide range of opinions about "involving patients in decisions," ranging from protective paternalism ("doctor knows best"), through enlightened self interest (lightening the load), to the potential rewards of a more egalitarian relationship with patients. The work points to three contextual precursors for the process: the availability of reliable information, appropriate timing of the decision making process, and the readiness of patients to accept an active role in their own management.

Conclusions Sharing decisions entails sharing the uncertainties about the outcomes of medical processes and involves exposing the fact that data are often unavailable or not known; this can cause anxiety to both patient and clinician. Movement towards further patient involvement will depend on both the skills and the attitudes of professionals, and this work shows the steps that need to be taken if further progress is to be made in this direction.
\end{abstract}

\section{Introduction}

Involving patients in decision making is becoming an important clinical task, ${ }^{12}$ particularly in general practice, where health professionals can guide patients before they enter domains in which treatment bias may operate. Sharing information is not the same as sharing decisions, ${ }^{3}$ and there is no evidence that the available models for involving patients in decision making are feasible or that doctors have the required skills. ${ }^{4}$ In broad terms, three models of doctor-patient interactionpaternalism, informed choice, and shared decision making-have been described and their inherent assumptions debated. ${ }^{5}$ A paternalistic approach involves

Towle's suggested steps for shared decision
making
- Develop a partnership with the patient
- Establish or review the patient's preference for
information-for example, amount and format
- Establish or review the patient's preferences for role
in decision making
- Ascertain and respond to patient's ideas, concerns,
and expectations
- Identify choices and evaluate the evidence from
research in relation to the individual patient
- Present (or direct to) evidence, taking into account
the above steps, and help the patient reflect on and
assess the impact of alternative decisions with regard
to his or her values and lifestyle
- Make or negotiate a decision in partnership, manage
conflict
- Agree on an action plan and complete
arrangements for follow up

taking the responsibility for decision making. Informed choice is at the opposite end of the spectrum, where the patient is provided with "sufficient" information and the clinician withdraws from the decision process. Shared decision making describes the middle ground. ${ }^{6}$ But exactly how the principle of "involving" patients resonates with practice has not been explored. ${ }^{78}$ Lists of competencies for involving patients have been proposed $^{9}{ }^{10}$ but not investigated.

It is therefore important to know if the theoretical constructs need to be adapted for use in clinical settings. We used focus groups to elicit the reactions of general practice registrars when they were asked to use a suggested model $^{9}$ in interactions with simulated patients in three specific disease areas (benign prostatic hypertrophy, menopausal symptoms, and atrial fibrillation). In contrast with one to one interviews, focus groups can explore differences in opinions as well as defining consensus and capitalise on group interaction to uncover hidden attitudes. ${ }^{12}{ }^{13}$

\section{Participants and methods}

Study sample

During 1998 four group interviews were held within the half day release sessions of vocational training schemes for general practice registrars in south Wales. Most researchers aim for homogeneity to gain peer group safety and the sample was purposefully selected to enable us to gauge the reactions of new general practitioners to the concept of involving patients in decision making. All the registrars attending three vocational training schemes in south Wales were invited to take part in the study.

\section{Interview structure}

Participants were introduced to the concept of sharing decisions with patients and provided with an outline of

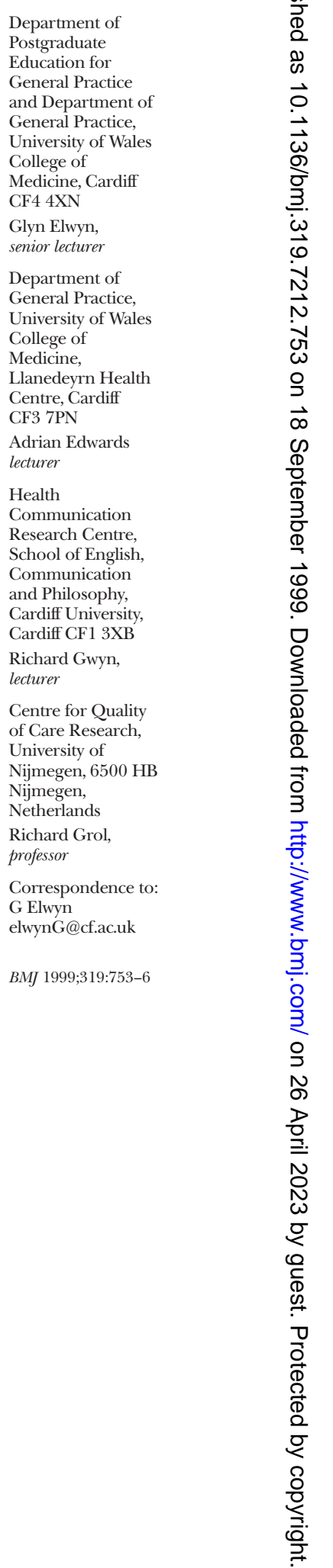


suggested stages $^{9}$ and a description of the clinical problems they would encounter. Three small sets (three or four people in each) were formed. Individuals volunteered in turn to consult with a simulated patient who had one of the three roles described in the box. The doctors were asked to conduct the discourse as if it were a "normal" consultation. The patients' roles and presentations were chosen, firstly, because they are typical of those seen in practice; secondly, because each clinical problem has treatment options that legitimately allow clinical equipoise-the patient's views can determine choice of treatment; and, thirdly, because systematic review data are available regarding the options. The simulated patients were non-medically trained individuals with previous experience in undergraduate training in communication skills.

Each set was assigned one clinical problem and the consultations conducted by different doctors in turn while others observed. Before the first consultation, a short description of the risks of each treatment option was provided. Before the second consultation numerical data about the risks were provided, and before the third consultation the same data were provided in a graphical format. This staged introduction enabled us to gauge the effect it had on the registrars' methods of involving patients and is reported separately. ${ }^{14}$

\section{Focus group interviews}

Group interviews were held after each consultation and reactions explored use of an interview schedule (see box). The simulated patients were present and given opportunities to contribute. The total duration of the interview was 80-90 minutes, and the proceedings were audiotaped and transcribed.

\section{Analysis}

The transcripts were examined by three authors (GE, $\mathrm{AE}, \mathrm{RGw})$ to identify emergent themes. ${ }^{11}$ These were agreed by discussion and the data categorised independently by two authors (GE and $\mathrm{AE}$ ), who subsequently agreed an overall classification. As our intention was to present viewpoints rather than achieve statistical generalisability, the data are not presented numerically. Trends and majority agreements, however, are indicated. The results were checked with the simulated patients and three of the course organisers and modified where required.

\section{Simulated patient roles}

The vignettes described clinical scenarios in which the problem has been identified so that the participants could concentrate entirely on the decision making aspect of the consultation:

Menopausal symptoms

Patient undecided about hormone replacement therapy and anxious about the risk of breast cancer

\section{Benign prostatic hypertrophy}

Patient wants to know more about the typical options that face a man who is told that he has "prostatism," with no other risk factors

Atrial fibrillation

Patient wants to know about the pros and cons of warfarin and aspirin for prevention of stroke

\section{Interview schedule}

Views on involving patients in decisions

- What do you think about the concept of "shared decision making"?

- Have you been trained in anything similar?

- Is it important to consider patients' preferences for "involvement"?

- What do you think about giving patients options?

Explore views on providing "data" to patients

- Does it help?

- How much information is useful?

- Is it feasible to do this within the diversity of general practice?

Explore views on the skills required

- What problems do you anticipate in practice?

- Are there groups of patients in whom this approach would be difficult or inappropriate?

- Are these inherent skills or do they need development?

\section{Results}

Of 45 registrars within the training schemes during the study period, $39(87 \%)$ attended one of four interviews in different parts of south Wales. Eight course organisers acted as observers, taking the total number of clinicians involved to 47 . Five themes were identified.

\section{Views about "shared decision making"}

Positive and negative views about involving patients All the participants agreed that the concept of "shared decision making" was novel-“it was new...doing something different from the talk we normally do"-and a spectrum of opinions was elicited. At one end was the view that no matter how data are presented it is unrealistic to expect patients to participate in decision making-"the patient has no information to make an informed choice. At the end of the day it is a professional judgment"; "they [the patients] haven't been to medical school for 5 years, how can we expect them to make a decision?"

There was also evidence of a professional reticence to undertake this approach-"I wouldn't have dreamed of showing you [the patient] the figures." Others were more receptive to the idea and the potential benefits of involving patients. This was thought to be particularly true when options were equally tenable-"it is more rewarding using them [the risk tools] because you feel you have informed the patient. They've got the information and have some part in the decision rather than just listen to us talking to them"; "[sharing decisions] unburdens the doctor...[when] there's a lot of uncertainty about what is the best thing to do."

\section{Barriers to sharing decisions}

Lack of information and a reluctance to share data-Most participants acknowledged the potential benefits, and discussions revolved around the difficulty of actually involving patients. It was said that sharing decisions “...is entirely content specific. You can't lay out options and their pros and cons if you don't know them"; "...threw into stark reality how often patients ask questions for which we don't have the information in the 
depth they require at our fingertips." Some thought that "specialists" might be better placed than generalists to take on this task. Nevertheless, there was agreement that patients want information in depth. All the participants agreed that data had to be robust-"it has got to be cast iron data."

Time and timing-Participants thought that it was very important to achieve the correct "timing" for shared decisions. In their opinion only a few consultations contain problems for which it is feasible to provide options. Decision making in their view should not be imposed on patients who are anxious and not ready to consider choices. Lack of time was cited as a barrier, particularly the time it would take to find accurate data, though this was not overemphasised. The view emerged that it is unusual for decisions to be taken within one consultation, so the task could be staged. Further discussions are often necessary and the agreed view was that "....sharing a decision is a process not an event."

Contextual modifiers-Many participants emphasised the need to be sensitive to "contextual" modifiers such as age and educational achievement. It was widely thought that some patients would have difficulty in understanding outcome data presented as probabilities. Presentations of choices, they said, often have to be simplified and at times omitted altogether. Participants were also conscious that established consulting patterns within a longstanding professional relationship could militate against the introduction of a new approach to decision making.

Types of decisions-Another obstacle was the nature of the decision itself. Sharing decisions was considered particularly appropriate in situations of professional equipoise about the "best" choice of treatment. It was thought that situations that lacked equipoise (such as urgent or dangerous medical problems) or situations of conflict (where patient "demand" is contrary to empirical evidence) needed different decision making approaches.

\section{Reported current practice}

When they were asked to compare these techniques against their "usual" practice most registrars stated that they normally bias their presentation of facts and consciously "steer" patients-"you choose the data to help the patient make the decision you think they ought to make. I'm sure I do that."

One doctor, talking about hormone replacement therapy, revealed a strategy of attempting to judge a patient's preferred choice before tailoring the data to reinforce the patient's view-"I try to establish what the patient really wants...then I push the information in that direction."

Some of the participants, however, were not prepared to allow patients into the decision making arena-"if the doctor feels that one course of treatment is better than another course of treatment, then that should be strongly pressed home."

There was also an unchallenged expression of irritation with the notion of the "informed patient," and data were viewed as a method of enforcing the doctor's decision-"they've come in after reading the damn patient leaflet and are worried about side effects. There's no way they can assess in their head what the risks are, so they just don't take it [the medication]"; "I spend a lot of my time telling people that they don't need whatever they've barged in and demanded...so statistics could be quite useful for that."

\section{Training and skill implications}

Although all the registrars had previously received training in communication skills, they all agreed that their previous experience of "...teaching had concentrated on the first part of the consultation. The emphasis has been on achieving rapport, matching agendas, and problem solving." Most participants were positive about the techniques being explored, which contrasted with their ambivalence about involving patients in decision making.

\section{Insights into the process of sharing decisions}

\section{Explicit about process}

The registrars thought that an essential feature of successful patient involvement was explicitness about the decision process and indicated that a useful way of legitimising patient involvement was by the use of phrases such as "this is a problem on which doctors do not have one view." Many registrars recounted that when the phrase "what would you like?" is used as a ploy to explore patient views, the typical response is, "I don't know, you're the doctor." There was general agreement about the need to develop methods of involving patients that seem neither insincere nor "rhetorical."

\section{Portrayal of options}

The participants noted that an important part of the process was a clear portrayal of choices. Some noted that they described options merely to undermine or dismiss them. Others noticed that they did not list all the options available - that there was a tendency not to describe the choice of "no action" or of deferring a decision.

\section{Patient role in decision making}

The doctors admitted that it was not their usual practice to ask patients about their preferred role in decision making. There was, however, an underlying assumption that most patients do want to be involved and that clinicians are good judges of their preferences-"I think there is this kind of intuitive judgment [about preferred role] that I often make when I first talk to a patient in the first part of the consultation."

\section{Opinions about possible "outcomes" of sharing decisions}

For many participants a positive outcome of sharing decisions was the increased sense of confidence that resulted from the feeling of being "protected by data." More commonplace in the discussion was the expression of concern about the potential anxiety in patients that could result from too much information and the added responsibility of decision making in the face of complex data about probabilities-"it's clear on an intuitive level that 'doctor uncertainty' is likely to distress a lot of patients"; "telling people about small risks will probably cause more trouble than keeping quiet until problems crop up."

The simulated patients suggested that other more patient oriented outcomes were important and placed a high value on the availability of choices and the perception of involvement in decision making. The 
patients thought that a key characteristic of a "successful" consultation would be the experience that information had been well presented and therefore understood, and they were confident that greater understanding would lead to a greater commitment to a chosen management option.

\section{Discussion}

Our exploratory work shows that this group of junior doctors had not developed the skills needed to involve patients in clinical decision making. These practitioners were in transition between the "hospital based" clinical environment and the culture of general practice; experienced doctors might react differently. The registrars were unaware of the benefits of patient participation in decision making and thought they did not have the information necessary to explain the risks and benefits of treatment choices. They admitted that "friendly persuasion" ${ }^{15}$ was their usual practice, justified on the grounds that the responsibility of being involved in decisions would lead to increased (and by implication unacceptable) anxiety in patients.

The use of simulated patients can be criticised for being one remove away from "actual" practice. ${ }^{16}$ Nevertheless, because our aim was to obtain views that were not based on abstract notions, this method was acceptable to the registrars and provided them with as close an experience as possible of the concepts of shared decision making within a peer group environment.

The stages of shared decision making suggested by Towle ${ }^{9}$ need modification to take into account the context, the type of decision, and the amount of control the patient prefers within the different stages of the interaction. ${ }^{10}$ Population based surveys cannot predict role preference ${ }^{17}$ and involvement needs to be tailored appropriately at every interaction. ${ }^{18}$ This work illustrates the complexity of achieving partnership with patients and the illogicality of asking patients about their preferred role until they have realised the possible harms and benefits entailed and their associated probabilities. Then, and only then, can it be legitimate to ask whether individuals want to take an active role in decision making. ${ }^{10}$

Our results show that practitioners need to adapt to varying contexts, preferences of patients, and types of decisions. ${ }^{19}$ To argue that patients should always be involved in clinical decisions is unwise. But as

\section{Stages and competencies of involving patients} in healthcare decisions ${ }^{10}$

1 Implicit or explicit involvement of patients in decision making process

2 Explore ideas, fears, and expectations of the problem and possible treatments

3 Portrayal of options

4 Identify preferred format and provide tailor made information

5 Checking process: understanding of information and reactions-for example, ideas, fears, and

expectations of possible options

6 Acceptance of process and preferred role in decision making

7 Make, discuss, or defer decisions

8 Arrange follow up

\section{Key messages}

- Involvement of patients in decisions about their treatment or care is increasingly advocated

- Registrars in general practice report not receiving training in the skills needed for successful involvement of patients in decision making

- Attitudes towards involving patients range from being highly positive to being more circumspect

- Moves towards enhancing patient involvement in decision making will depend on developing both skills and attitudes of professionals

information becomes readily available to all, this work starts to identify some of the steps required to implement the process effectively.

We are grateful for the commitment of Pat Oliver, Iris McKenzie, and Allan Thomas, who were the simulated patients; also for the secretarial work of Claire Collins and Diane Thomas in transcribing tapes; for comments on the draft of this paper by Nigel Stott, Trish Greenhalgh, Paul Kinnersley, Sandra Carlisle, Sharon Caple, Roisin Pill, Simon Smail, Sian Koppel, Clare Wilkinson, and Michel Wensing.

Contributors: GE and AE initiated the research, conducted the qualitative analysis, and drafted the paper. RGw attended the interviews and participated in the data analysis. RGr advised the project and data analysis and edited the manuscript. GE and AE are guarantors.

Funding: No external funding.

Competing interests: None declared.

1 Smith R. All changed, changed utterly. BMJ 1998;316:1917-8.

2 Richards T. Partnership with patients. BMJ 1998;316:85-6.

3 Ong LML, de Haes JCJM, Hoos AM, Lammes FB. Doctor-patient communication: a review of the literature. Soc Sci Med 1995;40:903-18.

4 Elwyn G, Edwards A, Kinnersley P. Shared decision-making: the neglected second half of the consultation. Br J Gen Pract 1999;49:477-82.

5 Gafni A, Charles C, Whelan T. The physician-patient encounter: the physician as a perfect agent for the patient versus the informed decision-making model. Soc Sci Med 1998;47:347-54.

6 Charles C, Gafni A, Whelan T. Shared decision-making in the medical encounter: what does it mean? (Or it takes at least two to tango). Soc Sci Med 1997;44:681-92

7 Gwyn R, Elwyn G. When is a shared decision not (quite) a shared decision? Negotiating preferences in a general practice encounter. Soc Sci Med 1999;49:437-47.

8 Elwyn G, Gwyn R, Edwards AGK, Grol R. Is a "shared decision" feasible in a consultation for a viral upper respiratory tract infection: assessing the influence of patient expectations for antibiotics using discourse analysis. Health Expectations 1999;2:105.

9 Towle A. Physician and patient communication skills: competencies for informed shared decision-making. Vancouver, Canada: University of British Columbia, 1997.

10 Elwyn G, Edwards A, Kinnersley P, Grol R. Shared decision-making: defining the competencies of involving patients in healthcare choices. Cardiff: Department of General Practice, University of Wales College of Medicine, 1999.

11 Frankland J, Bloor M. Some issues arising in the systematic analysis of focus group materials. In: Barbour R, Kitzinger J, eds. The use of focus groups in health research. London: Sage, 1997:144-55.

12 Kreuger R. Focus groups: a practical guide for research. London: Sage, 1988. 13 Morgan D. Focus groups as qualitative research. London: Sage, 1988.

14 Edwards A, Elwyn G, Gwyn R. General practice registrar responses to the use of different risk communication tools in simulated consultations: a focus group study. BMJ 1999;319:749-52.

15 Fisher S, Todd AD. Friendly persuasion: negotiating decisions to use oral contraceptives. In: Fisher S, Todd AD, eds. Discourse and institutional activity. New Jersey: Ablex Publishing, 1986.

16 Kinnersley P, Pill R. Potential of using simulated patients to study the performance of general practitioners. Br J Gen Pract 1993;43:297-300.

17 Guadagnoli E, Ward P. Patient participation in decision-making. Soc Sci Med 1998;47:329-39.

18 Dowie J. Decision analysis in guideline development and clinical practice: the "clinical guidance tree." In: Selbmann H-K, ed. Guidelines in health care. WHO conference report. Baden Baden: Nomos, 1998.

19 Edwards A, Matthews E, Pill RM, Bloor M. Communication about risk: diversity among primary care professionals. Fam Pract 1998;15:296-300. (Accepted 5 July 1999) 